\title{
Development of underwater microrobot with biomimetic locomotion
}

doi:10.1533/abbi.2006.0033

\author{
W. Zhang 1 , S. Guo 2,3 and K. Asaka ${ }^{4}$ \\ ${ }^{1}$ Graduate School of Engineering, Kagama University, Takamatsu, 761-0396 Japan \\ ${ }^{2}$ Faculty of Engineering, Kagama University, Takamatsu, Japan \\ ${ }^{3}$ Harbin Engineering University, People's Republic of China \\ ${ }^{4}$ Kansai Research Institute, AIST, 1-8-31 Midorigaoka, Ikeda, Osaka 563-8577, Japan
}

\begin{abstract}
Microrobots have powerful applications in biomedical and naval fields. They should have a compact structure, be easy to manufacture, have efficient locomotion, be driven by low voltage and have a simple control system. To meet these purposes, inspired by the leg of stick insects, we designed a novel type of microrobot with biomimetic locomotion with 1-DOF (degree of freedom) legs. The locomotion includes two ionic conducting polymer film (ICPF) actuators to realize the 2-DOF motion. We developed several microrobots with this locomotion. Firstly, we review a microrobot, named Walker-1, with 1-DOF motion. And then a new microrobot, named Walker-2, utilizing six ICPF actuators, with 3-DOF motion is introduced. It is $47 \mathrm{~mm}$ in diameter and $8 \mathrm{~mm}$ in height (in static state). It has $0.61 \mathrm{~g}$ of dried weight. We compared the two microrobot prototypes, and the result shows that Walker-2 has some advantages, such as more flexible moving motion, good balance, less water resistance, more load-carrying ability and so on. We also compared it with some insect-inspired microrobots and some microrobots with 1-DOF legs, and the result shows that a microrobot with this novel type of locomotion has some advantages. Its structure has fewer actuators and joints, a simpler control system and is compact. The ICPF actuator decides that it can be driven by low voltage (less than $5 \mathrm{~V}$ ) and move in water. A microrobot with this locomotion has powerful applications in biomedical and naval fields.
\end{abstract}

Key words: Biomimetic leg, insect-inspired robot, ICPF actuator, underwater microrobot.

\section{INTRODUCTION}

The demand for microrobots has increased in various fields of research. The research on underwater microrobots is one such field, for example, for cleaning the micropipeline in a radiating pattern, collecting samples from the seabed for archaeology or mining, scanning blood vessels for medical holography and so on.

The traditional motor has limitations in this field, because its electromagnetic structure is difficult to shrink to a compact volume. Smart materials, such as ionic conducting polymer film (ICPF), piezoelectric elements, braided pneumatic actuators, and shape memory alloys, which can be used as artificial muscles, paved the way for a large vari-

Corresponding Author:

W. Zhang

Graduate School of Engineering

Kagawa University, 2217-20, Hayashi-cho, Takamatsu 761-0396, Japan

Tel: +81-87-864-2356; Fax: +81-87-864-2369

Email: s04d506@stmail.eng.kagawa-u.ac.jp ety in microrobot design. A common characteristic of these kinds of actuators is that they can realize a fore-and-aft trajectory, instead of a rotating motion. The question arises, how to use the artificial muscles to design microrobots? Numerous scientists have focused on animals. Insects have been widely researched, for example, cockroaches, crickets, stick insects, ants and so on. Based on the results of these research studies, many insect-inspired robots with artificial muscles have been developed.

To develop a microrobot having fewer number of actuators, a compact structure, a simple control method, the ability to move in water and run on low voltage, we designed a novel type of locomotion using ICPF actuators.

An ICPF actuator consists of a perfluorosulfonic acid membrane with chemically plated gold as electrodes on both sides. It bends when a low voltage is applied between the electrodes (Hirose et al. 1992; Osada et al. 1992; Segalman et al. 1992; Oguro et al. 1993). The actuator is soft and can work in water or a wet environment. The ICPF actuator has several advantages. It runs on low voltage (above $1 \mathrm{~V})$, bends silently, responds quickly, consumes little energy, is environmentally friendly and has a long life. Its 


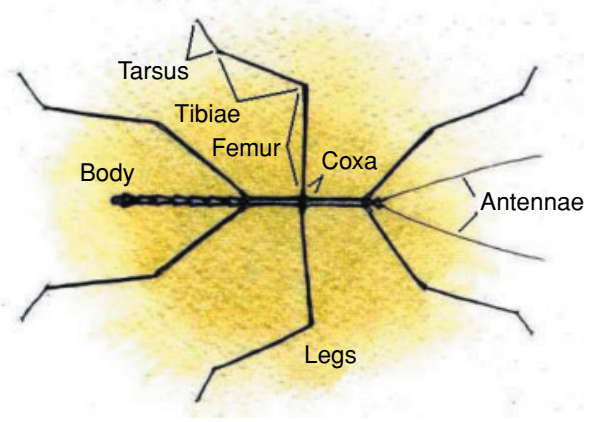

Figure 1 A sketch map of the stick insect.

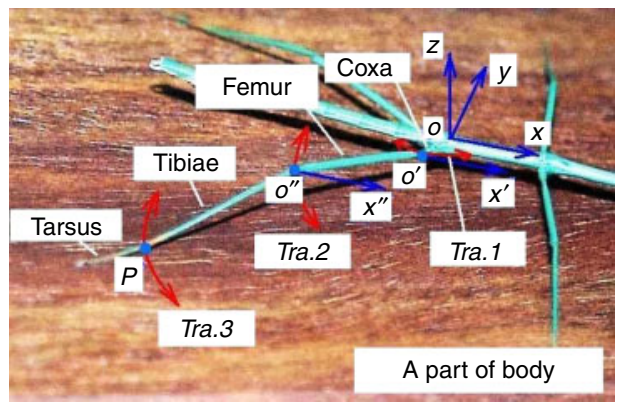

Figure 2 A sketch map of the trajectory variables of one stick insect leg.

density is close to that of water. The electromagnetic field of the ICPF actuator is practically undetectable. Propulsion using ICPF actuators generates a conspicuous wake.

During the last decade, ICPF actuators have been widely studied, and several kind of underwater microrobots utilizing ICPF actuators have been developed. ICPF actuators are used as artificial muscles to drive robots (Mojarrad and Shahinpoor 1997; Shahinpoor et al. 1998). Because of its quick response, the ICPF actuator is widely used as oscillating or undulating fins in swimming microrobots. Underwater microrobots with two tails have been developed (Guo et al. 1998, 2000, 2002, 2003, 2004; Laurent and Piat 2001). A prototype of a fish-like underwater robot with two pectoral fins has also been reported (Anton et al. 2004). A wireless microrobot that mimicks a tadpole has also been developed (Jung et al. 2003). But these kinds of swimming microrobots cannot locate a spot accurately. Hence, the development of a microrobot with a ground locomotion system has also been an area of focus by scientists. ICPF actuators are also used in biped walking underwater robot (Guo et al. 2004; Kamamichi et al. 2003). An ICPF micro-leg with 2 DOF (degree of freedom) has been developed (Otis et al. 2003). A ciliary motion-based eight-legged walking microrobot has also been developed (Ryu et al. 2002; Kim et al. 2003). But it seems that robots with these types of locomotion have some disadvantages; for example, they cannot resist the friction of the ground, have a complex control method or are less efficient movers.

Inspired by stick insects, we developed several microrobots incorporating a novel type of locomotion design

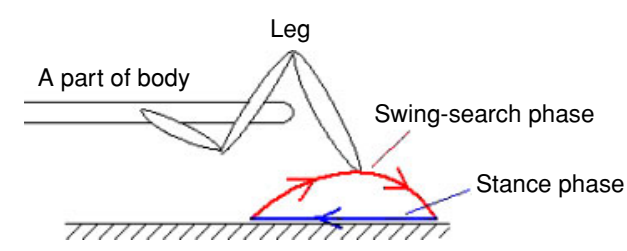

Figure 3 The two main phases in stick insect walking.

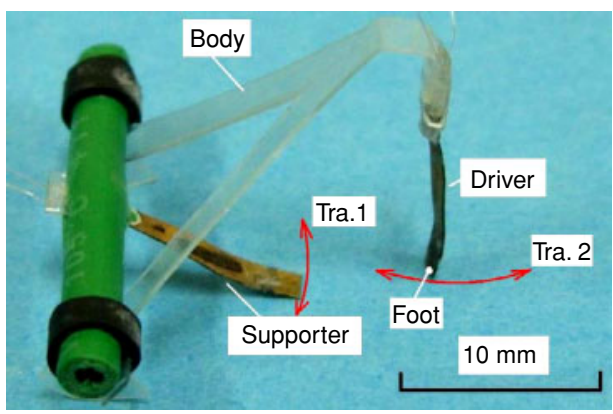

Figure 4 The structure of the biomimetic leg.

using ICPF actuators. Experimental results indicate that robots with this kind of locomotion have a good speed (Zhang and Guo 2005; Zhang et al. 2005a, 2005b).

This paper consists of four parts. Firstly, we review the locomotion of stick insects and introduce the improved biomimetic locomotion. Secondly, we review a microrobot and introduce a new microrobot. Thirdly, the characteristics of the new microrobot are introduced and compared with those of its predecessor. Lastly, we conclude with the results of our research.

\section{THE BIOMIMETIC LOCOMOTION WITH ICPF ACTUATORS}

\section{The review of the stick insect leg}

The structure of the stick insect is shown in Figure 1. Basically, each leg is composed of the coxa, the femur, the tibiae and the tarsus. Trajectory variables of one stick insect leg are shown in Figure 2. The coxa moves the point $o^{\prime}$ around the $z$-axis in trajectory Tra.1. The femur and the tibiae move points $\mathrm{o}^{\prime \prime}$ and $\mathrm{p}$ around the $x^{\prime}$ and $x^{\prime \prime}$ axes in Tra.2 and Tra.3, respectively. The tarsus does not contribute to the movements (Dean 1989). The coxa offers the foot 1-DOF motion in the direction of movement. The femur and the tibiae offer the foot a 2-DOF motion to enable it to find a reliable foothold together in the swingsearch phase and touch the ground and support the body while moving in the stance phase, as shown in Figure 3 (Cruse 1985a, 1985b).

\section{A biomimetic locomotion prototype}

Inspired by stick insects, a biomimetic locomotion prototype using two ICPF actuators has been designed, and is shown in Figure 4. It has three parts, two actuators and a body. The actuator in the vertical direction is called the 


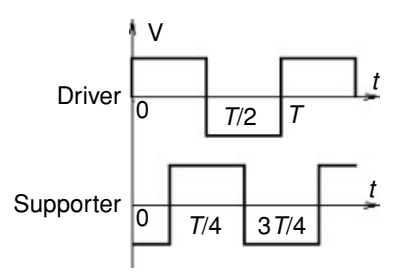

Figure 5 The control signals of the normal strategy. (a)

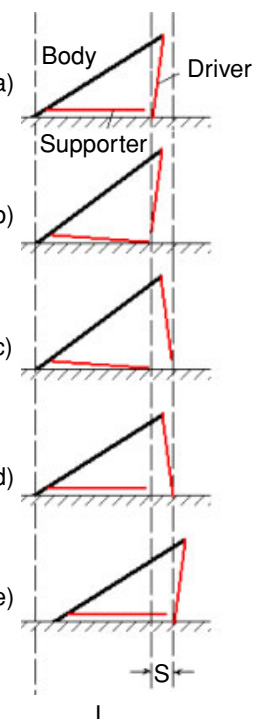

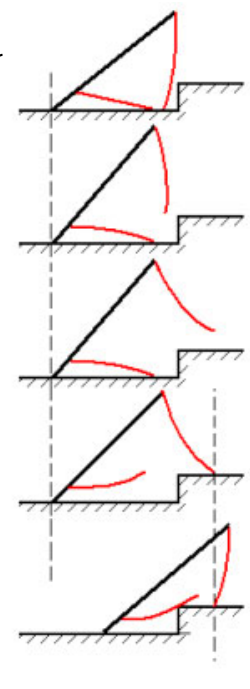

II

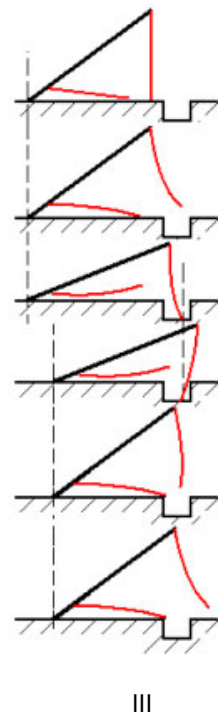

III
Figure 6 Different strategies for different environments.

driver. The free end of the driver is the foot. The driver offers the foot 1-DOF motion in the horizontal direction by bending along the trajectory Tra. 2 so that propulsion is offered. The actuator in the horizontal direction is called the supporter, and it offers the foot 1-DOF motion in the vertical direction by bending along the trajectory Tra.1.

Both the actuators are controlled by two signals. The control signals are shown in Figure 5. One-step circle is separated into four periods. Different states between two periods are shown in Figure 6(I), where the swing-search phase is from (a) to (d) and the stance phase is from (d) to (e). Strategies for different terrains are shown in Figure 6(II) and (III).

\section{A MICROROBOT WITH BIOMIMETIC LOCOMOTION}

\section{Review of a predecessor microrobot}

We had previously developed a microrobot, named Walker-1, shown in Figure 7 (Zhang et al. in press). The six ICPF actuators on plastic film body are divided into two groups. From $A$ to $C$ are the drivers and from $D$ to $F$ are the supporters.

Walker-1 can realize the walking and floating motion. With the use of control signals, as shown in Figure 5, it can walk in a straight line. Figure 8 shows one-step cycle, where the stance phase is from states (a)-(c) the swingsearch phase is from (c)-(e).

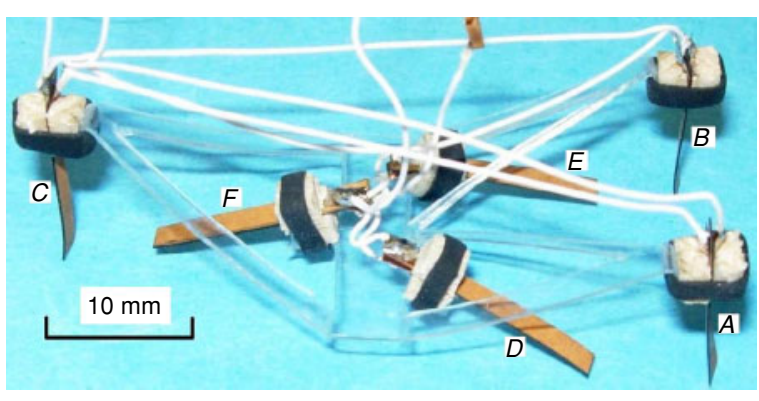

Figure 7 Outline of Walker-1. (a)

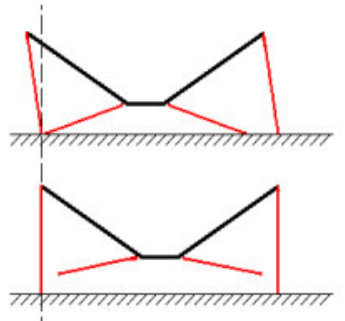

(c)

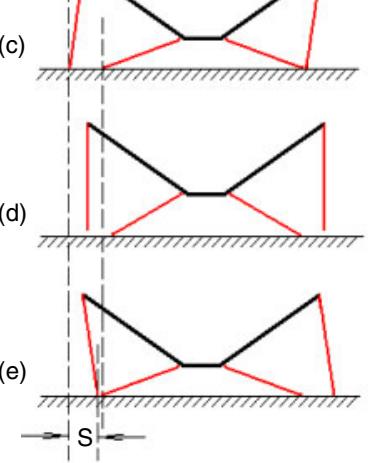

I

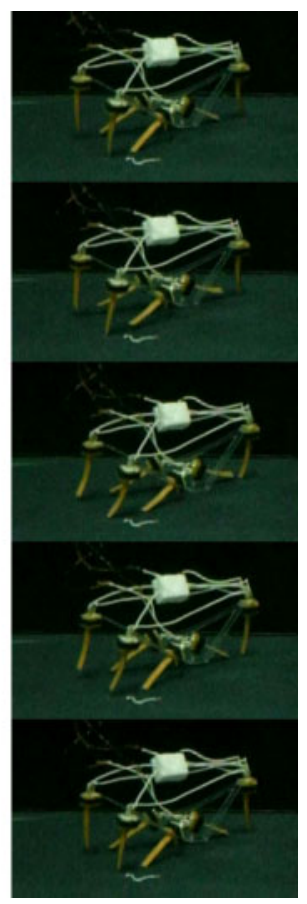

II
Figure 8 Walker-1 realized the two biomimetic phases.
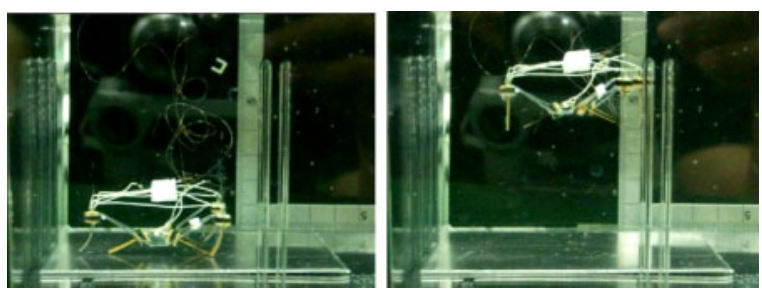

Figure 9 Two views of the floating motion experiment.

Decreasing the frequency to $0.3 \mathrm{~Hz}$ and electrolysing water can change the floatage. In this way, the microrobot can be controlled floating up and stop, as shown in Figure 9.

\section{The structure of the improved microrobot}

In our experiment on Walker-1, we found that it has some disadvantages. For example, it overturns easily and turns around hardly and so on. To overcome these disadvantages, an improved microrobot, named Walker-2, was developed, shown in Figures 10 and 11. 


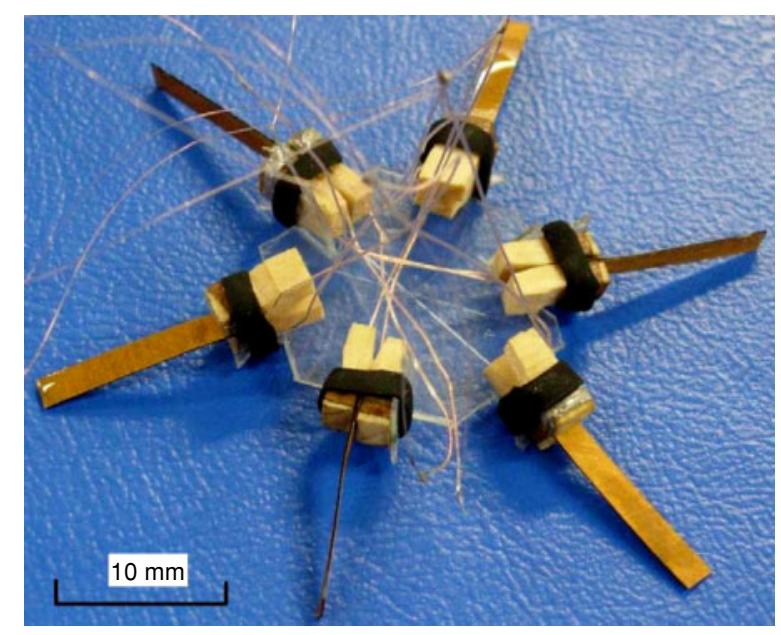

Figure 10 The outline of Walker-2.

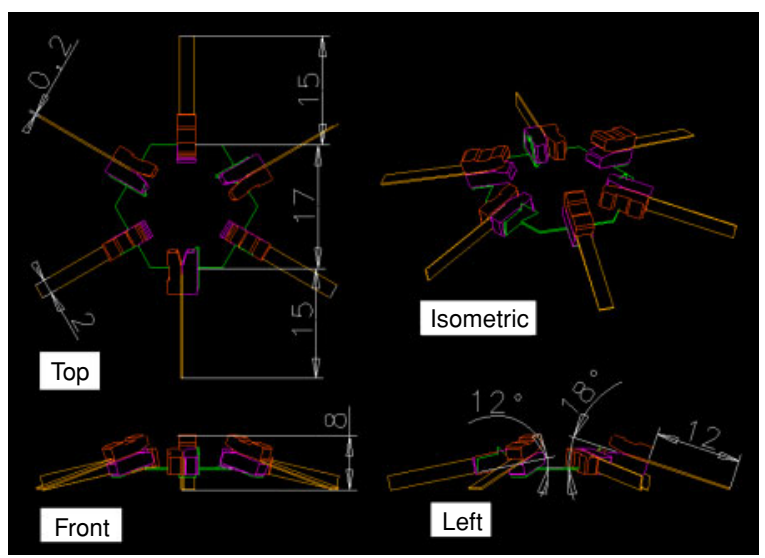

Figure 11 Dimensions of Walker-2.

Walker-2 is centrosymmetric with dimensions of $47 \mathrm{~mm}$ in diameter and $8 \mathrm{~mm}$ in height (in the static state). Its dried weight is $0.61 \mathrm{~g}$. Walker-2 has six ICPF actuators, with dimensions of $12 \mathrm{~mm} \times 2 \mathrm{~mm} \times 0.2 \mathrm{~mm}$, which are divided into three drivers and three supporters. The drivers and the supporters are symmetrically alternated around a hexagon film body in a radiating pattern.

\section{Mechanism and speed model of the walking motion}

Using different control signals, Walker-2 can move and rotate in the stance phase. Figure 12 shows a top view of Walker-2, where $A, B$ and $C$ stand for the drivers. The control strategies are shown in Table 1.

For example, direction 1 describes the forward motion. A step cycle is divided into four periods, as shown in Figure 13.

1. Period $A$, from states (a) to (b). In this period, the supporters lift the body up and the drivers are lifted away from the ground.

2. Period $B$, from states (b) to (c). In this period, two of the drivers bend to forward.

3. Period $C$, from states (c) to (d). In this period, the supporters bend upward enough so that the drivers can hold

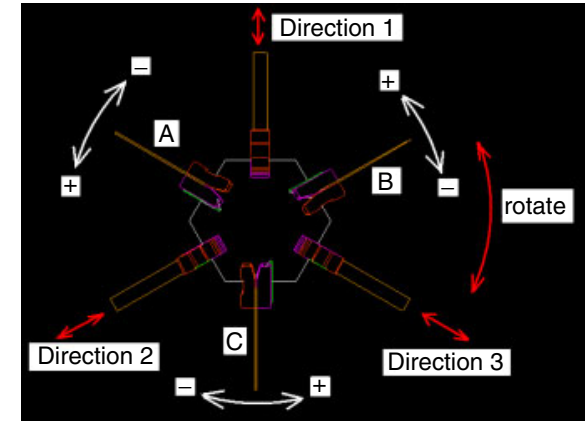

Figure 12 The DOFs of Walker-2.

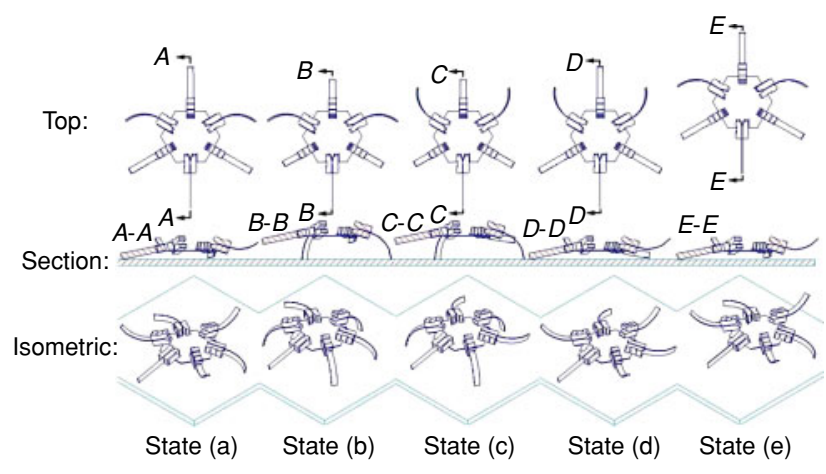

Figure 13 One-step cycle of forward motion of the robot.

Table 1 The control strategies of Walker-2

\begin{tabular}{lccc}
\hline & A & B & C \\
\hline Direction 1 forward & + & - & 0 \\
Direction 1 backward & - & + & 0 \\
Direction 2 forward & - & 0 & + \\
Direction 2 backward & + & 0 & - \\
Direction 3 forward & 0 & + & - \\
Direction 3 backward & 0 & - & + \\
Rotate clockwise & + & + & + \\
Rotate counter clockwise & - & - & - \\
\hline
\end{tabular}

'+' and '-' stand for the drivers' bending directions, and ' 0 ' means the driver without moving in stance phase.

the ground and keep the body until the supporters are away from the ground to avoid friction.

4. Period $D$, from states (d) to (e). In this period, the drivers bend in a direction contrary to that of the propulsion stroke, and the body is pushed forward.

The swing-search phase is from states (a) to (d). During this phase, the drivers are lifted away from the ground to find another foothold with the help of supporters. The stance phase is from states (d) to (e). In this phase, the drivers push the body forward while moving. The experimental video samples can be seen at http://www.weizhang.info-research-Walker-2.

The speed of the walking motion is decided by the displacement of the drivers and the frequency of the control signals, as shown in Figure 15 (left). Assume that the displacement of the actuator is $d$, and the microrobot moves 


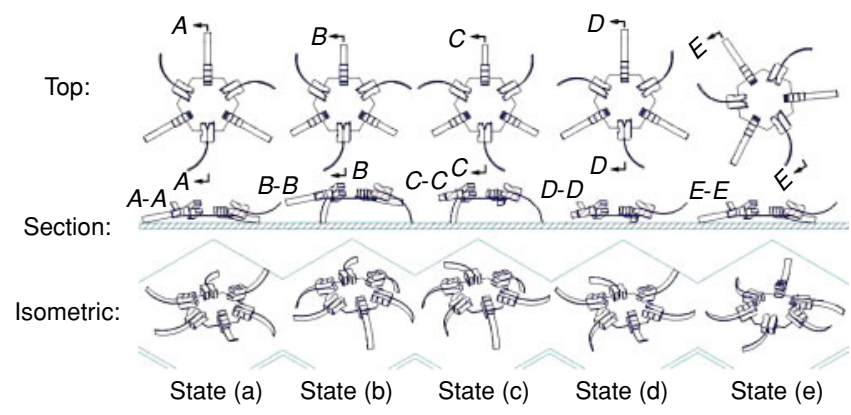

Figure 14 One-step cycle of rotating motion of the robot.
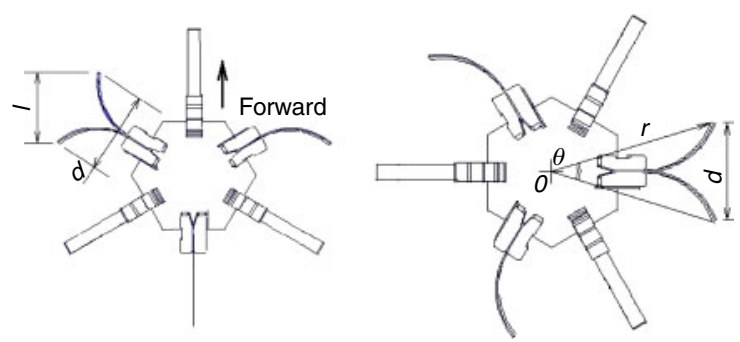

Figure 15 Left: The efficiency of the driver in walking motion. Right: The efficiency of the driver in rotating motion.

forward $l$ in one-step cycle, Equation (1) shows the relationship between $d$ and $l$. Equation (2) presents a speed model, where $v$ is the speed and $f$ is the frequency of the drivers' control signal.

$$
\begin{aligned}
& l=\frac{\sqrt{3}}{2} d \\
& v=\frac{\sqrt{3}}{2} d f .
\end{aligned}
$$

\section{Mechanism and the speed model of the rotating motions}

The centrosymmetric structure provides flexibility to Walker-2 during rotation. The rotating cycle is divided into four periods, as shown in Figure 14.

In brief, the swing-search phase is from states (a)-(d). In this period, the drivers prepare for the stroke with the help of supporters. The stance phase is from states (d) to (e). In this phase, the drivers push the rotating body.

The speed of rotation is decided by the displacement of the driver in the stance phase and the frequency of the step cycle, as shown in Figure 15 (right). The microrobot can rotate to an angle of $\theta$ in one-step cycle. So, it can be described by Equation (3), where $d$ is the displacement of the driver, $r$ is the radius of the robot and $2 r$ equals $47 \mathrm{~mm}$. Equation (4) represents the speed model, where $\omega$ and $f$ are the rotating speed and frequency, respectively.

$$
\begin{aligned}
& \theta=2 \arcsin \left(\frac{d}{2 r}\right) \\
& \omega=2 \arcsin \left(\frac{d}{2 r}\right) f .
\end{aligned}
$$

Table 2 The parameters for stability

\begin{tabular}{lll}
\hline & Walker-1 & Walker-2 \\
\hline The area among the drivers & $440 \mathrm{~mm}^{2}$ & $717 \mathrm{~mm}^{2}$ \\
The area among the supporters & $340 \mathrm{~mm}^{2}$ & $717 \mathrm{~mm}^{2}$ \\
$\begin{array}{l}\text { The height of the center of gravity } \\
\text { centre }\end{array}$ & $10 \mathrm{~mm}$ & $4 \mathrm{~mm}$ \\
\hline
\end{tabular}

Table 3 The DOFs of the robots

\begin{tabular}{lll}
\hline & Walker-1 & Walker-2 \\
\hline The DOFs on flat surface & 1 & 3 \\
The DOFs in vertical direction & OK & OK (theoretical) \\
\hline
\end{tabular}

Table 4 The area against the moving direction

\begin{tabular}{lll}
\hline & Walker-1 & Walker-2 \\
\hline The area that caused water resistance & $196 \mathrm{~mm}^{2}$ & $78 \mathrm{~mm}^{2}$ \\
\hline
\end{tabular}

\section{CHARACTERISTICS OF WALKER-2}

As an improved prototype, Walker-2 has some advantages over its predecessor, Walker-1.

\section{Stable structure}

Since both robots have a symmetric structure, their centres of gravity lie on the symmetric axial or centre. The stability of the robot is decided by the base area and the height of the centre of gravity. The larger the base area, more stable is Walker-2. The lower the centre of gravity, more stable is Walker-2. On the basis of Table 2, we draw the conclusion that Walker-2 is more stable than Walker-1, which is also proved by our experiment.

\section{Agile motions}

The flexibility of the two robots is shown in Table 3 . Theoretically, Walker-2 can also float vertically as does Walker-1.

\section{Less water resistance}

Water resistance is mostly decided by the area against the direction of movement. Table 4 shows that Walker-2 has less water resistance. 


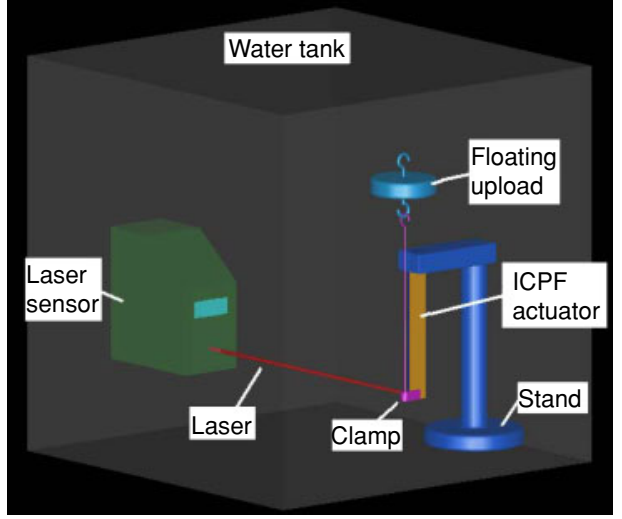

Figure 16 The upload ability measurement system simulating the driver's state in Walker-1.

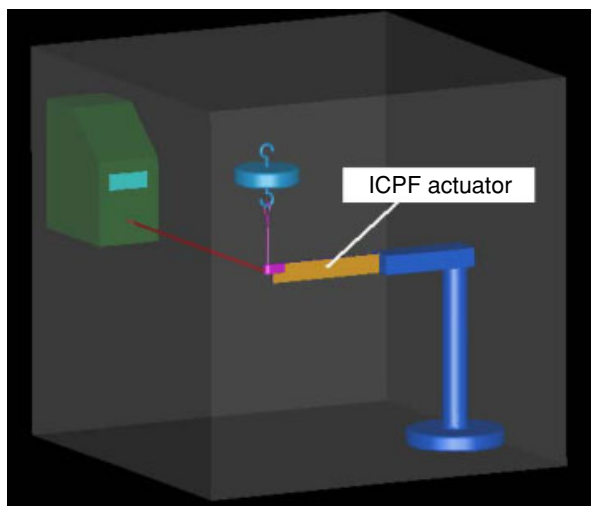

Figure 17 The upload ability measurement system simulating the driver's state in Walker-2.

\section{Powerful upload ability}

We simulated the state of the drivers in the two microrobots and measured their upload ability.

Because the pressure on the drivers acts in an upward direction in both microrobots, we designed a floating upload system to simulate the state of the drivers. Different states of the drivers are shown in Figures 16 and 17. In the system, an actuator is fixed at one end of an iron stand as a cantilever. A clamp with a hook is fixed at the tip of the actuator's free end. The weight of the clamp is balanced by its floatage, and so it can be ignored when we calculate the upload. The floating uploads are hooked on to the clamp. The floatage of each floating upload is $0.05 \mathrm{gf}$ in water. They can be used in series so as to gain different uploads. The system is set in a water tank. A laser sensor, placed outside the tank, measures the displacement at the free end of the actuator.

Without control signals, we measured the displacement by changing the floating uploads. Figure 18 describes the experimental result. Series 1 and series 2 are actuators' states in Walker-1 and Walker-2, respectively, as shown by Figures 16 and 17. With the same upload, series 2 has less displacement than do series 1 . The result indicates that

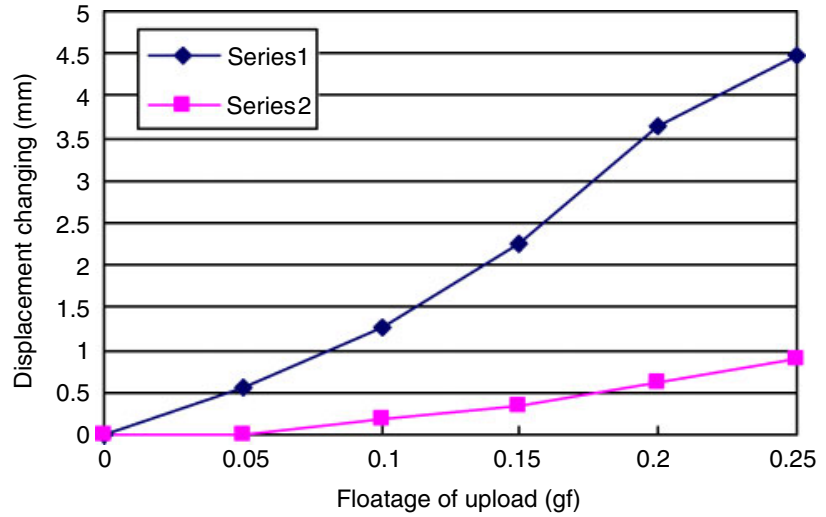

Figure 18 The flexibility of the static actuator.

the drivers' state in Walker-2 is stronger than in Walker-1, and it means that Walker-2 has more load-carrying ability.

\section{The disadvantage of Walker-2}

Limited by the structure, one driver is always in contact with the ground when the microrobot moves in a straight line. This driver resists the forward movement.

\section{CONCLUSIONS}

With this novel type of insect-inspired locomotion using ICPF actuators, we designed and developed an improved microrobot, Walker-2.

As we know, most of the insect-inspired robots use multi-DOF legs (Birch et al. 2000, 2001, 2002; Clark et al. 2001; Klaassen et al. 2002; Bachmann et al. 2002). These structures create some problems, such as the complex control strategy, too many wires for signal control or energy supply to robots' body, complex manufacturing, limitations in realizing a compact structure, high possibility of trouble and so on. A robot with the novel type of locomotion with 1-DOF legs can solve such problems.

With the advancement of manufacturing technology, microrobots with 1-DOF legs are widely being developed. A microrobot prototype and a solar-powered silicon robot with 1-DOF legs were developed (Kladitis et al. 1999; Hollar et al. 2003). It seems that they use dissymmetrical friction to move in a single direction, but the contrary friction produces a big resistance. A microrobot with eight 1-DOF ICPF legs and a walking silicon microrobot have been developed using the same principle (Ryu et al. 2002; Kim et al. 2003; Ebefors et al. 1999). The 1-DOF legs are divided into two groups to avoid friction. But because of its mechanical structure they can only realize 1-DOF motion. An obstacle or a low stair is a big problem, and multi-DOF motion is also a difficult mission for a robot with 1-DOF legs locomotion. In our work, we divided 1-DOF legs into two groups, the divers and supporters. In this way, the microrobots realize the biomimetic gait, avoid friction or tackle small obstacles and multi-DOF motion skillfully. 
In future, we will deal with the control system of microrobots.

\section{REFERENCES}

Anton M, Punning A, Aabloo A, et al. 2004. Towards a biomimetic EAP robot. In Proceedings of Towards the Autonomous Mobile Robots (TAROS2004), pp. 1-7.

Bachmann RJ, Kingsley DA, Quinn RD, et al. 2002. A cockroach robot with artificial muscles. In Proceedings of Climbing and Walking Robots Conference (CLAWAR02), 25-27 September 2002, Paris, France, pp. 659-66.

Birch MC, Quinn RD, Hahm G, et al. 2000. Design of a cricket microrobot. In Proceedings of IEEE International Conference on Robotics and Automation, April 2000, San Francisco, CA, vol. 2, pp. 1109-14.

Birch MC, Quinn RD, Hahm G, et al. 2001. A miniature hybrid robot propelled by legs. In Proceedings of the 2001 IEEE/RSJ International Conference on Intelligent Robots and Systems, vol. 2, pp. 845-51.

Birch MC, Quinn RD, Hahm G, et al. 2002. A Miniature Hybrid Robot Propelled by Legs. IEEE Robot Autom Mag, 9, pp. 20-30.

Clark JE, Cham JG, Bailey SA, et al. 2001. Biomimetic design and fabrication of a hexapedal running robot. In Proceedings of IEEE International Conference on Robotics and Automation, vol. 4, pp. 3643-9.

Cruse H. 1985a. Which parameters control the leg movement of a walking insect?, Part I: Velocity control during the stance phase. 7 Exp Biol, 116: 343-55.

Cruse H. 1985b. Which parameters control the leg movement of a walking insect?, Part II: The start of the swing phase. $\mathcal{F}$ Exp Biol, 116: 357-62.

Dean J. 1989. Leg coordination in the stick insect Carausius Morosus: Effects of cutting thoracic connectives. 7 Exp Biol, 145: 103-31.

Ebefors T, Mattsson JU, Kalvesten E, et al. 1999. A walking silison micro-robot. In the 10th International Conference on Solid-State Sensors and Actuators (Transducers'99), 7-10 June, 1999, Sendai, Japan, pp. 1202-5.

Guo S, Fukuda T, Kato N, et al. 1998. Development of underwater micro robot using ICPF actuator. In Proceedings of IEEE International Conference on Robotics and Automation, pp. 1829-34.

Guo S, Sugimoto K, Hata S, et al. 2000. A new type of underwater fish-like micro robot. In Proceedings of IEEE International Conference on Intelligent Robotics and Systems, pp. 862-7.

Guo S, Fukuda T, Asaka K. 2002. Fish-like underwater micro robot with 3 DOF. In Proceedings of IEEE International Conference of Robotics and Automation, May 2002, pp. 738-43.

Guo S, Fukuda T, Asaka K. 2003. A new type of fish-like underwater microrobot. IEEE / ASME Trans Mechatronics, 8: 136-41.

Guo S, Okuda Y, Asaka K. 2004. Development of a novel type of underwater micro biped robot with multi DOF. In Proceedings of the 14th International Offshore and Polar Engineering Conference, vol. II, pp. 284-9.

Hirose Y, Shiga T, Okada A, et al. 1992. Gel actuators driven by an electric field. In Proceedings of the 3rd International Symposium on Micro Machine and Human Science, pp. 21-6.
Hollar S, Flynn A, Bellew C, et al. 2003. Solar powered $10 \mathrm{mg}$ silicon robot. In MEMS 2003, 19-23 January 2003, Kyoto, Japan, pp. 706-11.

Jung J, Kim B, Tak Y, et al. 2003. Undulatory tadpole robot (TadRob) using ionic polymer metal composite (IPMC) actuator. In Proceedings of the IEEE/RSJ International Conference on Intelligent Robots and Systems, pp. 2133-8.

Kamamichi N, Kaneda Y, Yamakita M, et al. 2003. Biped walking of passive dynamic walker with IPMC linear actuator. In SICE Annual Conference, August 2003, Fukui, pp. 212-7.

Kim B, Ryu J, Jeong Y, et al. 2003. A ciliary based 8-legged walking micro robot using cast IPMC actuators. In Proceedings of IEEE International Conference on Robotics \& Automation, September 2003, vol. 3, pp. 2940-5.

Klaassen B, Linnemann R, Spenneberg D, et al. 2002. Biomimetic walking robot scorpion: Control and modeling. In Proceedings of the ASME Design Engineering Technical Conference, vol. 5, pp. 1105-12.

Kladitis PE, Bright VM, Harsh KF, et al. 1999. Prototype microrobots for micro positioning in a manufacturing process and micro unmanned vehicles. In Proceedings of IEEE MEMS'99, 17-21 January 1999, Orlando, FL, USA, pp. $570-5$.

Laurent G, Piat E. 2001. Efficiency of swimming microrobots using ionic polymer metal composite actuators. In Proceedings of IEEE International Conference on Robotics \& Automation, pp. 3914-9.

Mojarrad M, Shahinpoor M. 1997. Biomimetic robot propulsion using polymeric artificial muscles. In Proceedings of IEEE International Conference on Robotics and Automation, pp. 2152-7.

Oguro K, Asaka K, Takenaka H. 1993. Polymer film actuator driven by a low voltage. In Proceedings of 4th International Symposium Micro Machine and Human Science, Japan, pp. $39-40$.

Osada Y, Okuzaki H, Hori H. 1992. A polymer gel of electrically driven moiety. Nature, 355: 242-4.

Otis M, Bernier R, Pasco Y, et al. 2003. Development of an hexapod BioMicroRobot with Nafion-Pt IPMC microlegs. In Proceedings of the 25th Annual International Conference of the IEEE EMBS, September 2003, pp. 3423-6.

Ryu J, Jeong Y, Tak Y, et al. 2002. A ciliary motion based 8-legged walking micro robot using cast IPMC actuators. In International Symposium on Micromechatronics and Human Science, pp. 85-91.

Segalman DJ, Witkowski WR, Adolf DB, et al. 1992. Theory and application of electrically controlled polymeric gels. Smart Mater Struct, 1(1), article 015.

Shahinpoor M, Bar-Cohen Y, Simpson JO, et al. 1998. Ionic polymer-metal composites (IPMCs) as biomimetic sensors, actuators and artificial muscles-A review. Smart Mater Struct, 7(6):R15-30(1).

Tadokoro S, Yamagami S, Takamori T. 2000. An actuator model of ICPF for robotic applications on the basis of physicochemical hypotheses. In Proceedings of IEEE International Conference on Robotics and Automation, pp. 1340-6.

Zhang W, Guo S. 2005. The development of a new kind of underwater walking robot. In Proceedings of International Conference on Complex Medical Engineering, pp. 199-204.

Zhang W, Guo S, Asaka K. 2005a. A novel type of underwater crawling microrobot. In Proceedings of IEEE International Conference on Robotics and Biomimetics, pp. 155-60. 
Zhang W, Guo S, Asaka K. 2005b. Developments of two novel types of underwater crawling microrobots. In Proceedings of IEEE International Conference on Mechatronics and Automation, July 2005, pp. 1884-9.

Zhang W, Guo S, Asaka K. 2006. Design and experimental results of a tripodic biomimetic microrobot with 5 DOFs. In The 6th World Congress on Intelligent Control and Automation
(WCICA2006), 21-23 June 2006, Dalian, People's Republic of China, pp. 8378-82.

Zhang W, Guo S, Asaka K. in press. A Tripodic biomimetic underwater microrobots utilizing ICPF actuators. In Proceedings of IEEE/RSJ International Conference on Intelligent Robots and Systems, 9-15 October 2006, Beijing, People's Republic of China. 

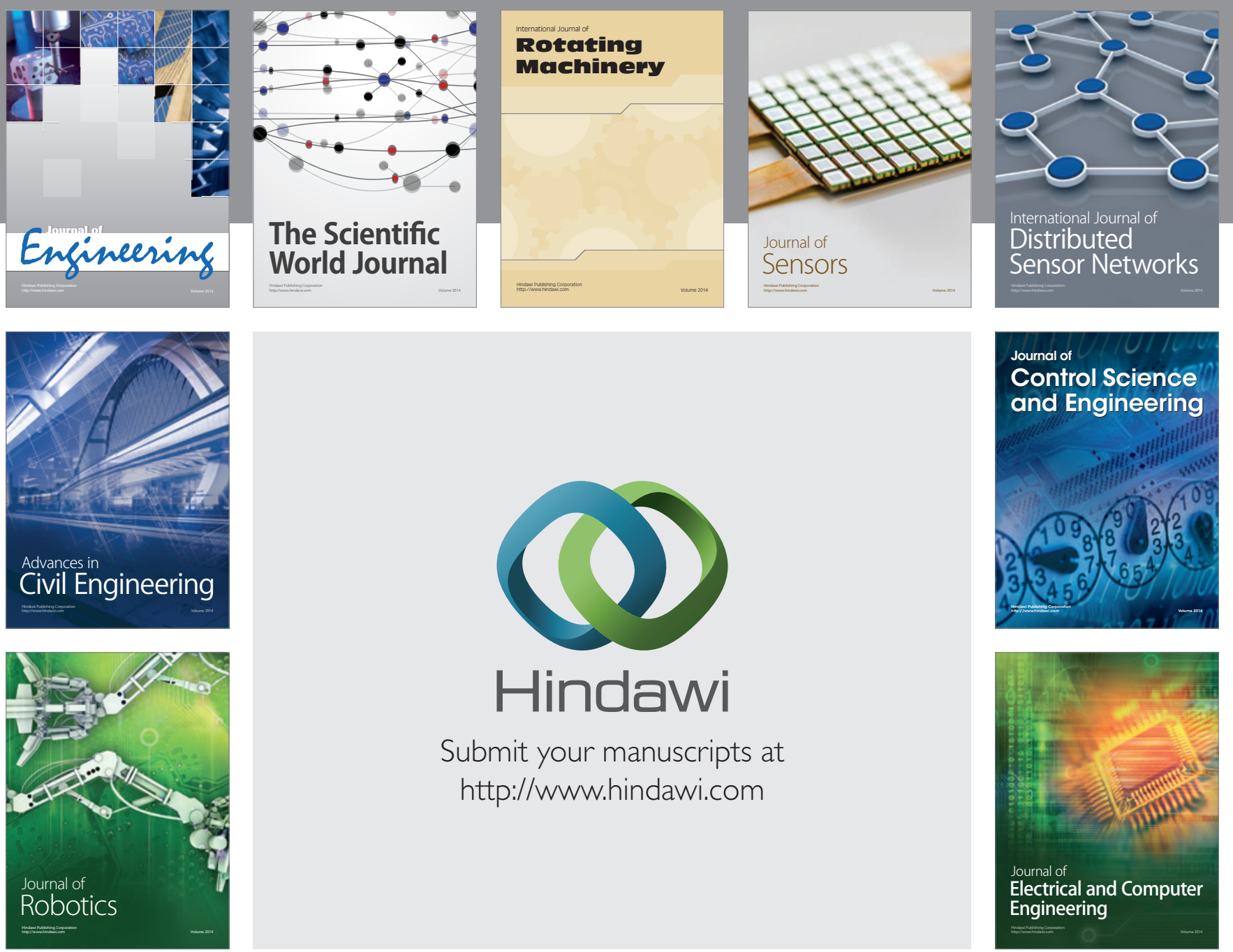

Submit your manuscripts at

http://www.hindawi.com
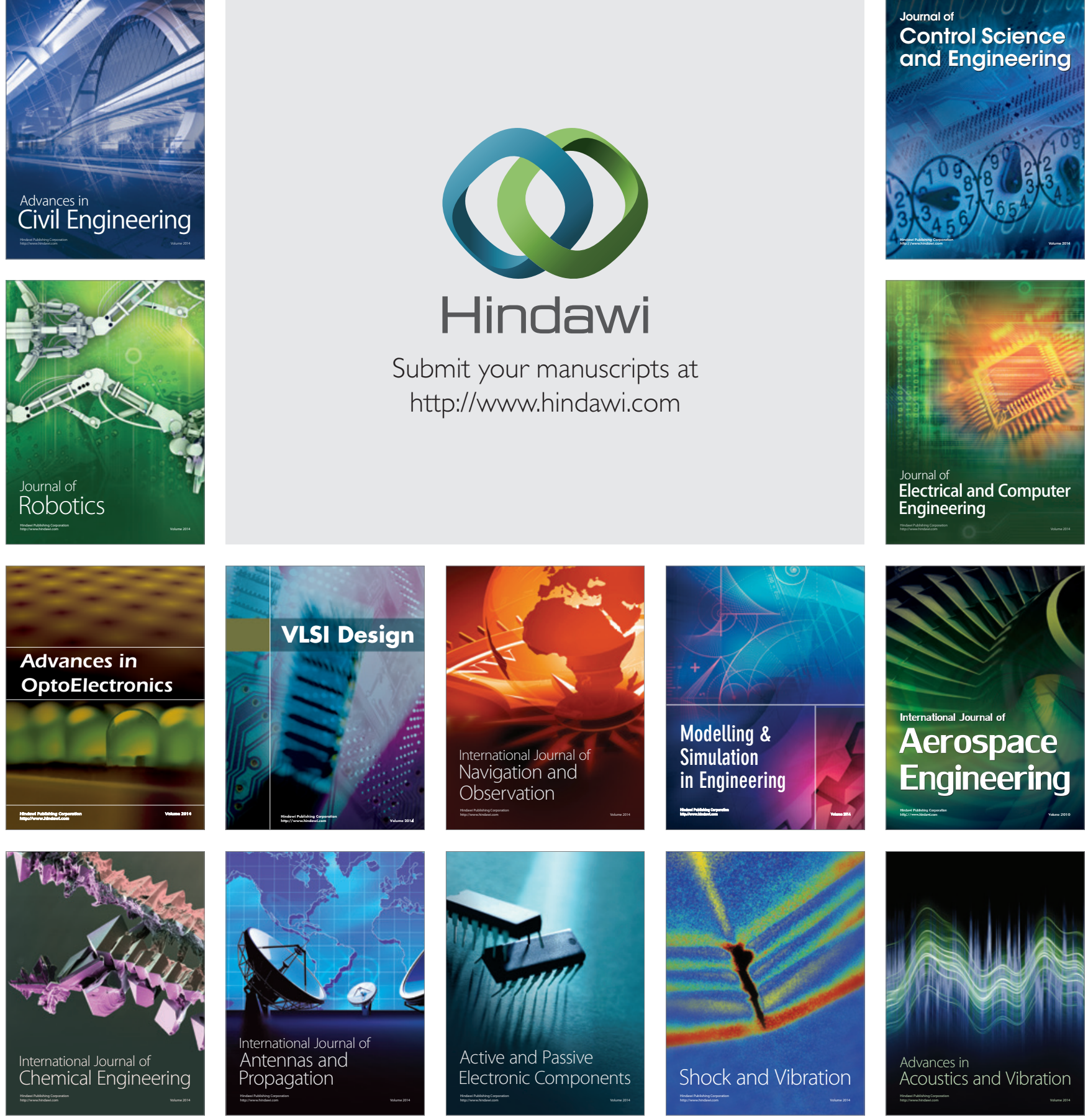